7:347-360.

3. Fukaura, H., et al. 1996. Induction of circulating myelin basic protein and proteolipid protein-specific transforming growth factor-beta1-secreting Th3 $\mathrm{T}$ cells by oral administration of myelin in multiple sclerosis patients. J. Clin. Invest. 98:70-77.

4. Karachunski, P.I., Ostlie, N.S., Okita, D.K., and Conti-Fine, B.M. 1997. Prevention of experimental myasthenia gravis by nasal administration of synthetic acetylcholine receptor $\mathrm{T}$ epitope sequences. J. Clin. Invest. 100:3027-3035.

5. Santos, L.M., al-Sabbagh, A., Londono, A., and Weiner, H.L. 1994. Oral tolerance to myelin basic protein induces regulatory TGF-beta-secreting T cells in Peyer's patches of SJL mice. Cell. Immunol. 157:439-447

6. Streilein, J.W. 2003. Ocular immune privilege: therapeutic opportunities from an experiment of nature. Nat. Rev. Immunol. 3:879-889.

7. Brown, B.D., et al. 2007. A microRNA-regulated lentiviral vector mediates stable correction of hemophilia B mice. Blood. 110:4144-4152.

8. Cerullo, V., et al. 2007. Antigen-specific tolerance of human alpha1-antitrypsin induced by helper-dependent adenovirus. Hum. Gene Ther. 18:1215-1224.

9. Dobrzynski, E., and Herzog, R.W. 2005. Tolerance induction by viral in vivo gene transfer. Clin. Med. Res. 3:234-240.

10. Franco, L.M., et al. 2005. Evasion of immune responses to introduced human acid alpha-glucosidase by liver-restricted expression in glycogen storage disease type II. Mol. Ther. 12:876-884.

11. Herzog, R.W. 2005. Recent advances in hepatic gene transfer: more efficacy and less immunogenicity. Curr. Opin. Drug Discov. Devel. 8:199-206.

12. Mingozzi, F., et al. 2003. Induction of immune tolerance to coagulation factor IX antigen by in vivo hepatic gene transfer. J. Clin. Invest. 111:1347-1356.

13. Ziegler, R.J., et al. 2004. AAV2 vector harboring a liver-restricted promoter facilitates sustained expression of therapeutic levels of alpha-galactosidase $\mathrm{A}$ and the induction of immune tolerance in fabry mice. Mol. Ther. 9:231-240.

14. Cao, O., et al. 2007. Induction and role of regulatory CD4+CD25+ T cells in tolerance to the transgene product following hepatic in vivo gene transfer. Blood. 110:1132-1140

15. Cao, O., Furlan-Freguia, C., Arruda, V.R., and Herzog, R.W. 2007. Emerging role of regulatory T cells in gene transfer. Curr. Gene Ther. 7:381-390.

16. Dobrzynski, E., et al. 2004. Induction of antigenspecific CD4+ T-cell anergy and deletion by in vivo viral gene transfer. Blood. 104:969-977.

17. Dobrzynski, E., et al. 2006. Prevention of cytotoxic $\mathrm{T}$ lymphocyte responses to factor IX-expressing hepatocytes by gene transfer-induced regulatory T cells. Proc. Natl. Acad. Sci. U. S. A. 103:4592-4597.

18. Hoffman, B.E., et al. 2007. Muscle as a target for supplementary factor IX gene transfer. Hum. Gene Ther. 18:603-613.

19. Sun, B., et al. 2007. Enhanced response to enzyme replacement therapy in Pompe disease after the induction of immune tolerance. Am. J. Hum. Genet. 81:1042-1049.

20. Lüth, S., et al. 2008. Ectopic expression of neural autoantigen in mouse liver suppresses experimental autoimmune neuroinflammation by inducing antigen-specific Tregs. J. Clin. Invest. 118:3403-3410.

21. Mempel, T.R., et al. 2006. Regulatory T cells reversibly suppress cytotoxic $\mathrm{T}$ cell function independent of effector differentiation. Immunity. 25:129-141.

22. Knolle, P.A., and Gerken, G. 2000. Local control of the immune response in the liver. Immunol. Rev. 174:21-34.

23. Limmer, A., et al. 2000. Efficient presentation of exogenous antigen by liver endothelial cells to CD8 + $\mathrm{T}$ cells results in antigen-specific T-cell tolerance. Nat. Med. 6:1348-1354.

\title{
Toward a broadly protective influenza vaccine
}

\section{Peter C. Doherty ${ }^{1,2}$ and Anne Kelso ${ }^{3}$}

1Department of Microbiology and Immunology, University of Melbourne, Melbourne, Victoria, Australia. 2Department of Immunology, St. Jude Children's Research Hospital, Memphis, Tennessee, USA. ${ }^{3}$ WHO Collaborating Centre for Reference and Research on Influenza, Parkville, Victoria, Australia.

\begin{abstract}
The current inactivated influenza virus vaccines induce antibodies that protect against closely related virus strains. They do not, however, protect against antibody-escape variants of seasonal influenza A viruses or new pandemic influenza A viruses emerging from non-human reservoirs. Might boosting influenza A virus-specific $\mathrm{CD8}^{+} \mathrm{T}$ cell memory diminish the danger posed by these variant viruses? Pre-existing $\mathrm{CD8}^{+} \mathrm{T}$ cell-mediated immunity directed at peptides from conserved internal proteins of the influenza $A$ virus does not prevent infection, but it can promote early virus clearance and decrease morbidity in mice. In this issue of the JCI, Lee et al. show that people who have not been exposed to avian influenza A (H5N1) viruses have cross-reactive $\mathrm{CD8}^{+} \mathrm{T}$ cell memory to a wide range of $\mathrm{H} 5 \mathrm{~N} 1$ peptides (see the related article beginning on page 3478 ). These peptides could be used to add a CD8 ${ }^{+}$ $T$ cell component to current antibody-focused vaccine strategies with a view to reducing the impact of infection with novel influenza A viruses.
\end{abstract}

The recent spread of the extremely virulent avian influenza A subtype H5N1 viruses, herein referred to as $\mathrm{H} 5 \mathrm{~N} 1$, through Asia and to North Africa and Europe has raised serious concerns about the possibility of a novel human influenza pandemic $(1,2)$. Though the severe disease that can develop

Nonstandard abbreviations used: M1, matrix protein 1; NA, neuraminidase; NP, nucleoprotein.

Conflict of interest: The authors have declared that no conflict of interest exists.

Citation for this article: J. Clin. Invest. 118:3273-3275 (2008). doi:10.1172/JCI37232. in humans exposed to H5N1-infected birds is rare and sustained human-to-human transmission of the virus has not yet been observed, the three influenza pandemics of the 20th century were all caused by influenza A viruses that originated from birds (3). Variant influenza A (H1N1), A (H3N2), and $\mathrm{B}$ viruses also cause regular seasonal epidemics that are associated with substantial morbidity and economic loss. It is bad enough that some 250,000-500,000 (particularly elderly) people die annually from influenza, but what if we should face an event like the 1918-1919 influenza pandemic? That pan- demic killed in excess of 40 million people worldwide - before the era of rapid air travel and at a time when the global population was less than a third of that today.

\section{Limitations of current influenza vaccines}

Inactivated influenza vaccines elicit neutralizing antibody responses that provide reasonable protection against the homologous H1N1, H3N2, and B viruses (4). However, antibody-mediated selection drives changes (known as antigenic drift) in the viral HA $(\mathrm{H})$ and neuraminidase (NA; N) surface glycoproteins, which in turn dictate the frequent production of a new vaccine, sometimes as often as annually, as has been the case in each of the last five years. The WHO recommends candidate vaccine virus strains that have been identified among the collections of emerging field isolates supplied by a global network of $124 \mathrm{WHO}$ National Influenza Centers and other diagnostic laboratories and characterized by the four WHO Collaborating Centers for Influenza (in London, Atlanta, Melbourne, and Tokyo). The WHO's recommendations also inform the composition of the live vaccines produced from "cold-adapted" viruses that 


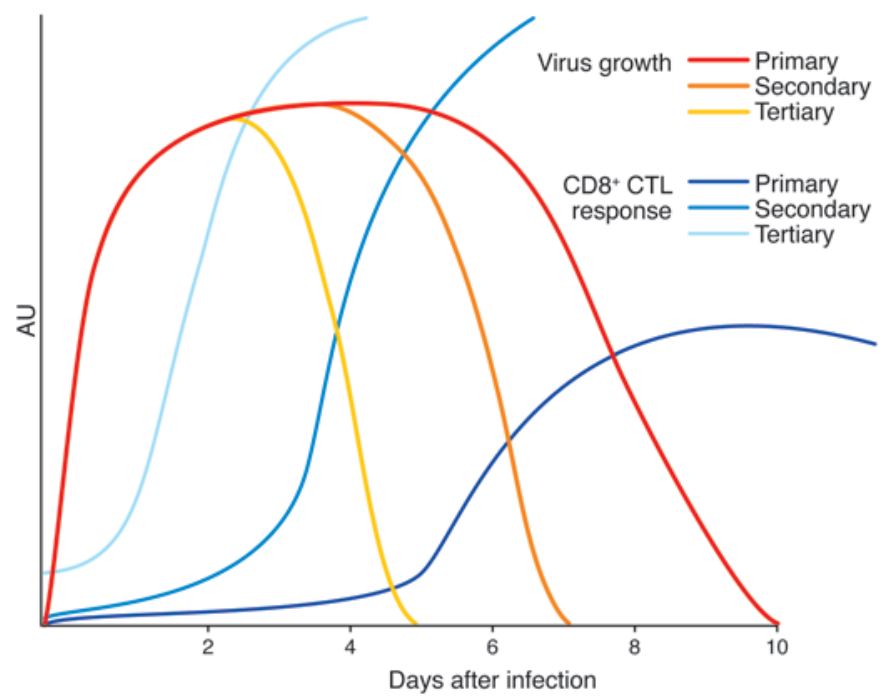

Figure 1

The CTL response to influenza A virus infection. Influenza A viruses rapidly grow to very high titers in the lungs of infected mice (primary virus growth). Virus clearance is only enhanced (secondary virus growth) by approximately 2-3 days (21) in those animals that have memory CD8 ${ }^{+}$CTL numbers at what might be considered normal, physiological prevalence $(<0.5 \%$ in spleen). Boosting those CTL counts (to $>5 \%$ ) a few weeks before viral challenge by some form of secondary stimulation can cause the period before the virus is successfully eliminated to be shortened by 48 hours or more (tertiary virus growth) (22). As shown in this issue of the $\mathrm{JCl}$ in the study by Lee et al. (14), most people already possess memory CTLs specific for the influenza A viruses. In the face of a rapidly emerging seasonal influenza epidemic, or a pandemic caused by a novel influenza A virus, a possible future strategy to mitigate the impact would be to stockpile a vaccine for emergency use that increases CD8+ CTL numbers.

are selected to grow at the lower temperature of the human nose and upper respiratory tract. These vaccines have been used for many years in Russia and the former Soviet Union and are now available internationally, but are not approved for Western use in the very young or the elderly.

Apart from the lack of protection against variant viruses, the use of traditional inactivated vaccines in a future influenza pandemic will be constrained by the need for specialized egg-based production facilities and long production times required for the creation of a new vaccine. Most influenza vaccines are made from viruses grown in the allantoic cavity of embryonated hen's eggs. The viruses are then inactivated, purified, and in most cases "split" to produce vaccines that consist largely of HA and NA subunits. Recent advances include the use of reverse genetics to express the HA and NA of extremely virulent viruses, such as $\mathrm{H} 5 \mathrm{~N} 1$, with the internal proteins of standard vaccine strains (5) in order to enable the virus to replicate without killing the chick embryo, as well as the development of cell culture systems for high-titer virus growth.
Even with these advances, however, it is likely to be many months before a new vaccine based on a pandemic virus can be deployed globally.

\section{Cross-protection by CTLs}

While nobody is suggesting that we abandon the current antibody-based strategy, there is increasing interest in the possibility that it might be useful to add a $\mathrm{CD}^{+}$ $\mathrm{T}$ cell-activating component to the trivalent seasonal influenza vaccines (6). The early finding $(7,8)$ that influenza A virusspecific CTLs are broadly cross-reactive for cells of the same MHC class I type infected with serologically distinct $\mathrm{H} 1 \mathrm{~N} 1$ and $\mathrm{H} 3 \mathrm{~N} 2$ viruses was initially greeted with scepticism, then by indifference. The argument is that if seasonal influenza infection does promote cross-reactive $\mathrm{T}$ cell responses, then why do so many people get sick every one or two years? The counter-argument is, of course, that the majority of individuals may be protected from more serious disease by their $\mathrm{T}$ cell response. Healthy adults do not usually die from influenza, and it is the very young, who have not previously been exposed to the virus, and the frail elderly who are particularly at risk. Adoptive T cell transfer or cross-priming experiments with $\mathrm{H} 1 \mathrm{~N} 1$ and $\mathrm{H} 3 \mathrm{~N} 2$ viruses in mice have demonstrated conclusively that established $\mathrm{CD}^{+} \mathrm{T}$ cell memory is protective (9). Primed mice may still show substantial weight loss and morbidity, but they clear serologically different influenza A viruses from the lung more rapidly than mice that are not primed and can even survive respiratory challenge with highly virulent H7N7 and $\mathrm{H} 5 \mathrm{~N} 1$ viruses (9).

The identity of the antigen recognized by influenza A virus-specific CTLs remained obscure for the best part of a decade until Alain Townsend and John Skehel in the United Kingdom and Jon Yewdell, Jack Bennink, Geoff Smith, and Bernie Moss at the NIH showed $(10,11)$ that the crossreactive CTLs generated in a number of mouse strains of different MHC class I types recognize the conserved internal influenza A virus nucleoprotein (NP). Subsequently, Townsend and colleagues broke open the CTL/MHC class I story by demonstrating that NP-specific $\mathrm{CD}^{+} \mathrm{T}$ cells recognize short viral peptides bound by the MHC class I molecules (12). This was a paradigmshifting experiment that led to the creation of the field of research on protein/peptide processing in the cytoplasmic compartment. Subsequently, a number of other internal proteins in the virus, particularly the acid and basic polymerases (PA and PB1, respectively) and the structural matrix protein 1 (M1), have been found to provide additional peptides that make up the peptide-MHC class I complexes recognized by influenza virus-specific CTLs in both mice and humans (13).

\section{The case for CTL-mediated cross-protective vaccines}

The article by Lee et al. in this issue of the JCI presents a detailed analysis, in individuals in the United Kingdom and Viet Nam, of the specificities of pre-existing $\mathrm{CD}^{+}$and $\mathrm{CD}^{+} \mathrm{T}$ cells for peptides encompassing all the proteins of an $\mathrm{H} 5 \mathrm{~N} 1$ and an $\mathrm{H} 3 \mathrm{~N} 2$ virus (14). Although citizens of the United Kingdom are not likely to have encountered an H5N1 virus, and the Vietnamese subjects were laboratory workers who were seronegative for $\mathrm{H} 5$, most subjects in both groups did possess memory $\mathrm{T}$ cells that were able to recognize cells displaying H5N1 peptides. A broad spectrum of peptides was recognized, predominantly from the viral NP and M1 proteins and, as subjects were not HLA typed, presumably reflecting a 
range of MHC class I-restriction specificities. Some, but by no means all, of these peptides were conserved between the H3N2 and H5N1 strains used.

Would it be worthwhile to boost this $\mathrm{T}$ cell memory in order to strengthen crossprotective immunity (Figure 1)? Though CD8 ${ }^{+}$CTL immunotherapy can control Epstein-Barr virus-induced lymphomagenesis in humans (15), experience with vaccines that function exclusively by promoting $\mathrm{CD}^{+} \mathrm{T}$ cell-mediated immunity in higher primates and humans has largely been restricted to the HIV and SIV lentiviruses. The results have generally been disappointing. Experiments with SIV vaccines have shown, for example, that the virus is controlled for a time following vaccination but then mutates to avoid $\mathrm{CD}^{+} \mathrm{T}$ cell surveillance (16). However, the situation with influenza A viruses is different, as these viruses neither integrate cDNA into the host genome nor persist in the host in any form. Enhanced control of influenza viruses in the short term is thus likely to be sufficient to limit disease and reduce transmission. Although expansion of the population of pre-existing memory $T$ cells may only serve to change the disease profile from mortality to morbidity, the impact could be substantial in the event of a pandemic or a severe seasonal epidemic.

What type of vaccine might be appropriate to induce cross-protective CTLs? Inactivated vaccines induce negligible $\mathrm{CD}^{+} \mathrm{T}$ cell responses, and live, cold-adapted vaccines can induce $\mathrm{CD}^{+} \mathrm{T}$ cell memory cells, but the numbers are low. For repeated administration, the most practicable possibilities would be the use of either DNA encoding, particularly, the whole M1 and NP proteins (17), or of a spectrum of immunogenic peptides from these and other viral components (18). Another approach would be to separate NP and M1 proteins made in the current vaccine production protocol and then to deliver them with an adjuvant or linker molecule to promote their entry into, and degradation via, the cytoplasmic processing pathway for presentation with MHC class I molecules. However, repeated use of viral proteins or viral vectors (e.g., vaccinia viruses or alphaviruses) has the problem that bound antibodies would tend to target the NP or M1 protein for lysosomal degradation so that they do not enter the cytoplasmic processing pathway. Whatever strategy is used, a major advantage of a broad-spectrum $\mathrm{CD}^{+} \mathrm{T}$ cell vaccine is its potential for largescale stockpiling prior to need.

Are there risks in giving regular doses of a vaccine that promotes $\mathrm{CD}^{+} \mathrm{T}$ cell responses? Some have expressed concern that primed $\mathrm{CD}^{+}{ }^{+}$and $\mathrm{CD} 8^{+} \mathrm{T}$ cells might contribute to the excessive immune response known as a cytokine storm (19), which can cause early mortality in those individuals infected with the H5N1 and (most likely) the H1N1 virus responsible for the 1918 influenza pandemic. However, as the analysis of this systemic shock phenomenon progresses, it appears that the chemokines and cytokines involved are produced by elements of the innate response, especially monocytes and neutrophils (20). The first goal must therefore be to remove the virus from the equation as soon as possible after infection, an effect that can be mediated by a rapidly emerging recalled $\mathrm{CD}^{+} \mathrm{T}$ cell response. All in all, as suggested by Lee et al. in their current study (14), the possibility of developing a vaccination strategy for the regular boosting of influenza $\mathrm{A}$ virus-specific $\mathrm{T}$ cell-mediated immunity would seem eminently worthy of further consideration and experiment.

\section{Acknowledgments}

The Melbourne WHO Collaborating Centre for Reference and Research on Influenza is supported by the Australian Government Department of Health and Ageing. P.C. Doherty is funded by the NIH, the Australian National Health and Medical Research Council, and The American Lebanese Syrian Associated Charities (ALSAC) at St. Jude Children's Research Hospital.

Address correspondence to: Peter C. Doherty, Department of Immunology, St. Jude Children's Research Hospital, 332 North Lauderdale, Memphis, Tennessee 38105, USA. Phone: (901) 495-3470; Fax: (901) 495-3107; E-mail: peter.doherty@ stjude.org or pcd@unimelb.edu.au.

\footnotetext{
1. Li, K.S., et al. 2004. Genesis of a highly pathogenic and potentially pandemic $\mathrm{H} 5 \mathrm{~N} 1$ influenza virus in eastern Asia. Nature. 430:209-213.

2. Russell, C.J., and Webster, R.G. 2005. The genesis of a pandemic influenza virus. Cell. 123:368-371.

3. Belshe, R.B. 2005. The origins of pandemic influenza - lessons from the 1918 virus. N. Engl. J. Med.
} 353:2209-2211.
4. Palese, P. 2006. Making better influenza virus vaccines? Emerg. Infect. Dis. 12:61-65.

5. Hoffmann, E., Krauss, S., Perez, D., Webby, R., and Webster, R.G. 2002. Eight-plasmid system for rapid generation of influenza virus vaccines. Vaccine. 20:3165-3170.

6. Epstein, S.L., et al. 2005. Protection against multiple influenza A subtypes by vaccination with highly conserved nucleoprotein. Vaccine. 23:5404-5410.

7. Effros, R.B., Doherty, P.C., Gerhard, W., and Bennink, J. 1977. Generation of both cross-reactive and virus-specific T-cell populations after immunization with serologically distinct influenza A viruses. J. Exp. Med. 145:557-568.

8. Zweerink, H.J., Askonas, B.A., Millican, D., Courtneidge, S.A., and Skehel, J.J. 1977. Cytotoxic T cells to type A influenza virus; viral hemagglutinin induces A-strain specificity while infected cells confer crossreactive cytotoxicity. Eur. J. Immunol. 7:630-635.

9. Thomas, P.G., Keating, R., Hulse-Post, D.J., and Doherty, P.C. 2006. Cell-mediated protection in influenza infection. Emerg. Infect. Dis. 12:48-54.

10. Townsend, A.R., and Skehel, J.J. 1984. The influenza A virus nucleoprotein gene controls the induction of both subtype specific and cross-reactive cytotoxic T cells. J. Exp. Med. 160:552-563.

11. Yewdell, J.W., Bennink, J.R., Smith, G.L., and Moss, B. 1985. Influenza A virus nucleoprotein is a major target antigen for cross-reactive anti-influenza A virus cytotoxic T lymphocytes. Proc. Natl. Acad. Sci. U. S. A. 82:1785-1789.

12. Townsend, A.R., et al. 1986. The epitopes of influenza nucleoprotein recognized by cytotoxic T lymphocytes can be defined with short synthetic peptides. Cell. 44:959-968.

13. Gotch, F., McMichael, A., Smith, G., and Moss, B. 1987. Identification of viral molecules recognized by influenza-specific human cytotoxic $\mathrm{T}$ lymphocytes. J. Exp. Med. 165:408-416.

14. Lee, L.Y.-H., et al. 2008. Memory T cells established by seasonal human influenza A infection crossreact with avian influenza $\mathrm{A}(\mathrm{H} 5 \mathrm{~N} 1)$ in healthy individuals. J. Clin. Invest. 118:3478-3490.

15. Rooney, C.M., et al. 1998. Infusion of cytotoxic T cells for the prevention and treatment of EpsteinBarr virus-induced lymphoma in allogeneic transplant recipients. Blood. 92:1549-1555.

16. Barouch, D.H., et al. 2002. Eventual AIDS vaccine failure in a rhesus monkey by viral escape from cytotoxic T lymphocytes. Nature. 415:335-339.

17. Epstein, S.L., et al. 2002. DNA vaccine expressing conserved influenza virus proteins protective against $\mathrm{H} 5 \mathrm{~N} 1$ challenge infection in mice. Emerg. Infect. Dis. 8:796-801.

18. Day, E.B., et al. 2007. The context of epitope presentation can influence functional quality of recalled influenza A virus-specific memory CD8+ T cells. J. Immunol. 179:2187-2194.

19. Cheung, C.Y., et al. 2002. Induction of proinflammatory cytokines in human macrophages by influenza A (H5N1) viruses: a mechanism for the unusual severity of human disease? Lancet. 360:1831-1837.

20. Szretter, K.J., et al. 2007. Role of host cytokine responses in the pathogenesis of avian $\mathrm{H} 5 \mathrm{~N} 1 \mathrm{influ}-$ enza viruses in mice. J. Virol. 81:2736-2744.

21. Flynn, K.J., Riberdy, J.M., Christensen, J.P., Altman, J.D., and Doherty, P.C. 1999. In vivo proliferation of naive and memory influenza-specific CD8(+) T cells. Proc. Natl. Acad. Sci. U. S. A. 96:8597-8602.

22. Christensen, J.P., Doherty, P.C., Branum, K.C., and Riberdy, J.M. 2000. Profound protection against respiratory challenge with a lethal $\mathrm{H} 7 \mathrm{~N} 7$ influenza $\mathrm{A}$ virus by increasing the magnitude of CD8 $(+)$ T-cell memory. J. Virol. 74:11690-11696. 


\title{
Tracing the molecular pathogenesis of antiphospholipid syndrome
}

\author{
Hartmut Weiler
}

Blood Research Institute, BloodCenter of Wisconsin, Milwaukee, Wisconsin, USA.

\begin{abstract}
Fetal loss induced by antiphospholipid antibodies (aPLs) in mice is a complement-driven inflammatory condition. Engagement of the complement receptor $\mathrm{C5aR}$ on neutrophils induces expression of the principal initiator of the blood clotting mechanism, tissue factor (TF), and blocking this downstream event of complement activation prevents antibodyinduced fetal loss. In this issue of the JCI, the study by Redecha et al. clarifies that in mice, the contribution of TF to this pathogenic mechanism is independent of its role in coagulation and thrombosis, but involves inflammatory signaling through the receptor PAR2 (see the related article beginning on page 3453 ). The study not only sheds light on a critical effector mechanism of aPL-induced fetal loss, but also suggests that treatment with statins, which decrease TF and PAR2 expression, may hold promise as a therapeutic approach to antiphospholipid syndrome-associated pregnancy complications.
\end{abstract}

Antiphospholipid syndrome (APS) is characterized by the presence of autoreactive antiphospholipid antibodies (aPLs), which recognize specific plasma proteins that possess an affinity for anionic phospholipids, combined with clinical evidence of thrombosis. In pregnant females, aPLs trigger severe pregnancy complications, such as miscarriage, intrauterine growth restriction, and fetal death, and also increase the likelihood of preeclampsia (1-3). Infusion of aPLs isolated from human patients into pregnant mice is sufficient to reproduce fetal loss and fetal growth restriction. A remarkable series of genetic and pharmacologic intervention studies in this mouse model has led to a detailed understanding of the pathogenesis underlying aPLinduced fetal loss $(1,4,5)$.

Clinically, the most relevant antigen recognized by aPLs is $\beta 2$-glycoprotein I $(\beta 2 \mathrm{GPI})$, a plasma protein with poorly characterized functions. Binding of aPL induces the formation of $\beta 2 \mathrm{GPI}$ dimers with increased affinity for anionic phospholipids. Binding to negatively charged mem-

Nonstandard abbreviations used: -a, activated; aPL, antiphospholipid antibody; APS, antiphospholipid syndrome; $\beta 2 \mathrm{GPI}, \beta 2$-glycoprotein I; C5aR, C5a receptor; FVII, Factor VII; PAR, protease-activated receptor; TF, tissue factor.

Conflict of interest: The author has declared that no conflict of interest exists.

Citation for this article: J. Clin. Invest. 118:3276-3278 (2008). doi:10.1172/JCI37243. brane surfaces is also essential for the function of key coagulation proteins, including prothrombin. Competition between aPL/ $\beta 2$ GPI complexes and coagulation factors for binding to phosphatidylserine explains the anticoagulant effect exerted by aPLs in in vitro coagulation assays. The affinity for negatively charged membranes directs the selective allocation of aPL/ $\beta 2$ GPI complexes to the phosphatidylserine-rich surface of fetal trophoblast cells in the placenta.

A critical milestone in understanding aPL-induced fetal loss was the observation of massive accumulation of complement component $\mathrm{C} 3$ in the placenta and the finding that that genetic deficiency of maternal C3 or administration of the C3 inhibitor Cryy-IgG prevents fetal loss (6). It has since been shown that downstream of C3 in this signaling pathway, interactions between complement component $\mathrm{C} 5 \mathrm{a}$ and the $\mathrm{C} 5 \mathrm{a}$ receptor $(\mathrm{C} 5 \mathrm{aR})$, rather than the classic cell-destroying formation of the C5b-C9 membrane attack complex, are responsible for fetal loss (7).

Thus far, 2 specific consequences of C5a-C5aR engagement are known to contribute to fetal loss: augmented release of TNF (8) and induction of tissue factor (TF) expression by maternal neutrophils (9). TNF- $\alpha$ may exert direct cytotoxic effects on trophoblast cells that line maternal blood spaces in the placenta, or sustain the activation of immune cells in the placenta. Accordingly, genetic ablation or functional inhibition of TNF- $\alpha$ and its interactions with its receptors prevents aPL-induced fetal loss (8). More recent work shows that the aPL-induced expression of TF on neutrophils is a critical second link between C5aR engagement and fetal loss (9). C5aRdeficient neutrophils fail to increase TF expression in response to aPL, and pharmacologic or genetic suppression of TF in maternal neutrophils is sufficient to prevent the formation of reactive oxygen species and fetal loss.

\section{TF mediates fetal loss independent of thrombosis}

In the study by Redecha et al. in this issue of the JCI (10), the authors distinguish the relative role of the 2 known functions of TF. TF is a transmembrane receptor for the circulating coagulation Factor VII (FVII) (11). Upon formation of the TF/ FVII complex, TF gains affinity for plasma coagulation Factor X (FX) and converts it - via partial proteolysis - from an inactive zymogen to the active protease form (FXa). After release from the ternary TF/FVIIa/ FXa complex, FXa assembles with FVa on negatively charged membranes to form the principal enzymatic complex (prothrombinase) driving the generation of thrombin. Formation of the ternary complex of TF, FVIIa, and FX is regulated at the level of TF expression as well as by the redox status of preformed TF (12). With few exceptions, constitutive TF expression is limited to cell types that are sequestered from access to blood and blood components by the endothelial cell barrier of blood vessels. Injury to the endothelial cell barrier facilitates physical contact of TF and FVII and thereby triggers the blood coagulation reaction. Inflammatory mediators compromise endothelial barrier function and induce expression of TF in endothelial cells and various immune cell populations. This activates blood coagulation at sites of inflammation. However, the TF/FVIIa/FXa complex not only initiates coagulation, but can also elicit cellular signaling processes via proteolytic activation of $\mathrm{G}$ pro- 